\title{
Singular integrals and potentials in some Banach function spaces with variable exponent
}

\author{
Vakhtang Kokilashvili and Stefan Samko \\ (Communicated by Alois Kufner)
}

2000 Mathematics Subject Classification. 42B20, 47B38.

Keywords and phrases. Banach function space, non-increasing rearrangement, variable exponent, singular integral operators, Riesz potential, Lyapunov curve, curve of bounded rotation.

\footnotetext{
Abstract. We introduce a new Banach function space - a Lorentz type space with variable exponent. In this space the boundedness of singular integral and potential type operators is established, including the weighted case. The variable exponent $p(t)$ is assumed to satisfy the logarithmic Dini condition and the exponent $\beta$ of the power weight $\omega(t)=|t|^{\beta}$ is related only to the value $p(0)$. The mapping properties of Cauchy singular integrals defined on Lyapunov curves and on curves of bounded rotation are also investigated within the framework of the introduced spaces.
}

\section{Introduction}

In the last decade the generalized Lebesgue spaces $L^{p(\cdot)}(\Omega)$ and the related Sobolev type spaces $W^{m, p(x)}\left(R^{n}\right)$ attracted much attention, we refer to Sharapudinov [26] (1979), [27] (1996), Kovácík , Rákosník [19] (1991), Edmunds, Rákosnik [10] (1992), Samko [24]-[23] (1998), [25] (1999), Edmunds, Lang, Nekvinda [9] (1999), which obviously grows at present, see for example, the recent investigations Cruz, Fiorenza, Neugebauer [3] 
(2002), Diening [5]-[6] (2002), Diening, Růžička [7] (2002), Edmunds, Nekvinda [8] (2002), Fiorenza [11](2002), Kokilashvili, Samko [15]-[17] (2002), and references therein. Investigations in this topic are strongly stimulated by applications in various problems related to objects with non-standard local growth in which growth conditions of variable order arise (in elasticity theory, fluid mechanics, differential equations, see for example Růžička [22], [7] and references therein). The spaces $L^{p(\cdot)}(\Omega)$ and $W^{m, p(x)}\left(R^{n}\right)$ proved to be an appropriate tool applicable in this area.

The theory of the spaces $L^{p(\cdot)}(\Omega)$ nowadays is quickly developed. After the first disappointment caused by some undesirable properties (functions from these spaces are not $p(x)$-mean continuous, the space $L^{p(\cdot)}(\Omega)$ is not translation invariant, convolution operators in general do not behave well and so on) a rapid progress followed for continuous exponents $p(x)$ satisfying the logarithmic Dini condition. We mention in particular the result on denseness of $C_{0}^{\infty}$-functions in the Sobolev space $W^{m, p(x)}\left(R^{n}\right)$, see [25], and the breakthrough connected with the study of maximal operators, see [5], [6].

Because of applications, a reconsideration of the main theorems of harmonic analysis is actual, with the aim to find new proofs of those theorems which remain valid for variable exponents, or to find their substituting analogs. Among the challenging problems there were: the Sobolev type theorem on boundedness of the Riesz potential operator $I^{\alpha}$ from $L^{p(\cdot)}$ into $L^{q(\cdot)}, \frac{1}{q(x)}=\frac{1}{p(x)}-\frac{\alpha}{n}$ and the boundedness in $L^{p(\cdot)}$ of singular integral operators. Boundedness of $I^{\alpha}$ (Sobolev type theorem) for bounded domains was proved in [23] conditionally, under the assumption that the maximal operator is bounded in the spaces $L^{p(\cdot)}$, which turns to be unconditional after the result of [5]-[6] on maximal operators (we refer also to [3] for maximal operators on unbounded domains).

Singular operators within the framework of the spaces with variable exponents were treated in [18], [17] and [7].

We introduce a new form of spaces with variable exponents for which the problem of boundedness of singular type integral operators may be resolved positively in a natural way, including the case of weighted spaces with variable exponents. We consider the Calderon-Zygmund operators, singular operators with the Cauchy kernel along Lyapunov curves or curves of bounded variation in the complex plane, the Riesz potential operator and the Poisson integral and its conjugates. The main statements are given in Theorems 3.1-3.5, 4.1-4.4.

\section{On some Banach function spaces}

Let $(\Omega, \mu)$ be a measure space and $M(\Omega, \mu)$ a space of measurable functions on $\Omega$. 
Definition 1. A normed linear space $X=\left(X(\Omega, \mu),\|\|_{X}\right)$ is called a Banach function space, if the following conditions are satisfied:

i) the norm $\|f\|_{X}$ is defined for all $f \in M(\Omega, \mu)$;

ii) $\|f\|_{X}=0$ if and only if $f(x)=0 \mu$-a.e. on $\Omega$;

iii) $\|f\|_{X}=\||f|\|_{X}$ for all $f \in X$;

iv) for every $Q \subset \Omega$ with $\mu Q<\infty$ we have $\left\|\chi_{Q}\right\|_{X}<\infty$;

v) if $f_{n} \in M(\Omega, \mu), n=1,2, \ldots$ and $f_{n} \nearrow f \mu$-a.e. on $\Omega$, then

$$
\left\|f_{n}\right\|_{X} \nearrow\|f\|_{X}
$$

vi) if $f, g \in M(\Omega, \mu)$ and $0 \leq f(x) \leq g(x) \mu$-a.e. on $\Omega$, then

$$
\|f\|_{X} \leq\|g\|_{X}
$$

vii) given $Q \subset \Omega$ with $\mu Q<\infty$, there exists a constant $c_{Q}$ such that for all $f \in X$,

$$
\int_{Q}|f(x)| d \mu \leq c_{Q}\|f\|_{X}
$$

Every Banach function space is a Banach space. For definition and fundamental properties of Banach function space we refer to [2].

We shall deal with some special Banach function space.

Let $\Omega$ be a bounded open subset of $R^{n}$ and $p(x)$ a measurable function on $\Omega$ such that

$$
1<p_{0} \leq p(x) \leq P<\infty, \quad x \in \bar{\Omega}
$$

and

$$
|p(x)-p(y)| \leq \frac{A}{\ln \frac{1}{|x-y|}},|x-y| \leq \frac{1}{2}, \quad x, y \in \bar{\Omega} .
$$

By $L^{p(\cdot)}(\Omega)$ we denote the space of measurable functions $f(x)$ on $\Omega$ such that

$$
A_{p}(f)=\int_{\Omega}|f(x)|^{p(x)} d x<\infty .
$$

This is a Banach function space with respect to the norm

$$
\|f\|_{L^{p(\cdot)}}=\inf \left\{\lambda>0: A_{p}(f / \lambda) \leq 1\right\}
$$

(see e.g. [9]). We denote

$$
\frac{1}{q(x)}=1-\frac{1}{p(x)} .
$$

The following integral transforms will be treated:

a) the potential operator

$$
I^{\alpha(x)} f(x)=\int_{\Omega} \frac{f(y)}{|x-y|^{n-\alpha(x)}} d x, \quad 0<\alpha(x)<n,
$$


b) the weighted potential operator

$$
I_{\beta}^{\alpha(x)} f(x)=\left|x-x_{0}\right|^{\beta} \int_{\Omega} \frac{f(y)}{\left|y-x_{0}\right|^{\beta}|x-y|^{n-\alpha(x)}} d y, \quad x_{0} \in \Omega
$$

and

c) the Hardy-type operator

$$
H^{\beta} f(x)=x^{\beta-1} \int_{0}^{x} \frac{f(t)}{t^{\beta}} d t, \quad H_{*}^{\beta} f(x)=x^{\beta} \int_{x}^{l} \frac{f(t)}{t^{\beta+1}} d t,
$$

where $0<\ell<\infty$.

In [16] (see also [15], [14] ) the following theorems were proved:

Theorem I. Under assumptions (1.1), (1.2) and the conditions

$$
\inf _{x \in \Omega} \alpha(x)>0 \text { and } \sup _{x \in \Omega} \alpha(x) p(x)<n,
$$

the potential operator $I^{\alpha(\cdot)}$ is bounded from $L^{p(\cdot)}(\Omega)$ into $L^{r(\cdot)}(\Omega)$ with $\frac{1}{r(x)}=\frac{1}{p(x)}-\frac{\alpha(x)}{n}$.

Theorem II. If the assumptions (1.1)-(1.2) and the condition $\inf _{x \in \Omega} \alpha(x)>$ 0 are satisfied, then the operator $I^{\alpha(\cdot)}$ is compact in $L^{p(\cdot)}(\Omega)$.

Theorem III. Under assumptions (1.1), (1.2) and the condition $\inf _{x \in \Omega} \alpha(x)>0$, the operator $I_{\beta}^{\alpha(\cdot)}$ is bounded in $L^{p(\cdot)}(\Omega)$ if

$$
-\frac{n}{p\left(x_{0}\right)}<\beta<\frac{n}{q\left(x_{0}\right)} \text {. }
$$

Theorem IV. Let $1 \leq p(x) \leq P<\infty$ for $x \in[0, \ell]$.

I. Let the conditions (1.1), (1.2) be satisfied on a neighbourhood $[0, d]$ of the origin, $d>0$. Then the operators $H^{\beta}$ and $H_{*}^{\beta}$ are bounded from $L^{p(\cdot)}(\Omega)$ into $L^{s(\cdot)}(\Omega)$ with any $s(x)$ such that $1 \leq s(x) \leq S<\infty$ for some $S, 0 \leq x \leq \ell$, and

$$
s(0)=p(0) \text { and }|s(x)-p(x)| \leq \frac{A}{\ln \frac{1}{x}}, 0<x \leq \delta, \quad 0<\delta<1,
$$

if

$$
-\frac{1}{p(0)}<\beta<\frac{1}{q(0)}
$$

II. If $p(0) \leq p(x), 0 \leq x \leq d$, for some $d>0$, then the same statement on boundedness from $L^{p(\cdot)}(\Omega)$ into $L^{s(\cdot)}(\Omega)$ is true if the requirement of validity 
of conditions (2.1), (2.2) on $[0, d]$ is replaced by the weaker assumption

$$
p(0)>1 \text { and }|p(x)-p(0)|<\frac{A}{\ln \frac{1}{x}}, \quad 0<x<\min (\ell, 1 / 2) .
$$

Observe that Theorem IV provides norm estimates for Hardy operators in spaces with variable exponent. In [28] there was proved a natural fact that the modular inequality for the Hardy operator (and more generally for some integral operators) is impossible in the case of variable exponents, see [28], Theorem 2.2.

On the base of $L^{p(\cdot)}$ we introduce now some new Banach function spaces. By

$$
f^{*}(t)=\sup \{s \geq 0: m\{x \in \Omega:|f(x)|>s\}>t\}
$$

we denote the non-increasing rearrangement of a function $f, m$ denoting the Lebesgue measure. It is clear that $f^{*}(t)=0$ for $t>m \Omega$, since $m \Omega<\infty$.

Let the function $p(t)$ satisfy condition (1.1) for $t \in[0, m \Omega]$. In the following definition we use the notation

$$
f^{* *}(t)=\frac{1}{t} \int_{0}^{t} f^{*}(y) d y, \quad f^{*}(t) \leq f^{* *}(t)
$$

Definition 2. The subset of all functions of $M(\Omega, m)$ for which

$$
\|f\|_{\Lambda^{p(\cdot)}}=\left\|f^{* *}\right\|_{L^{p(\cdot)}}<\infty
$$

is called the space $\Lambda^{p(\cdot)}$.

According to Theorem IV we conclude that there exists a constant $c>0$ such that

$$
\left\|f^{*}\right\|_{L^{p(\cdot)}} \leq\left\|f^{* *}\right\|_{L^{p(\cdot)}} \leq c\left\|f^{*}\right\|_{L^{p(\cdot)}} .
$$

Note that $\left\|f^{* *}\right\|_{L^{p(\cdot)}}$ is a norm. The triangle inequality follows from the inequality

(See e.g. [12], Section 2).

$$
(f+g)^{* *}(t) \leq f^{* *}(t)+g^{* *}(t) .
$$

Proposition 1. $\Lambda^{p(\cdot)}$ is a Banach function space.

Proof. Most of requirements of Definition 1 follow directly from properties of non-increasing rearrangements of functions and properties of the space $L^{p(\cdot)}$.

For example, iv) is valid since for $0 \leq f_{n} \nearrow f$ we have $f_{n}^{*} \nearrow f^{*}$ (see e.g. [29], Lemma 3.5, Chapter 5). Then

$$
\left\|f_{n}^{*}\right\|_{L^{p(\cdot)}} \nearrow\left\|f^{*}\right\|_{L^{p(\cdot)}}
$$

by the property of $L^{p(\cdot)}$. 
Applying the Hölder inequality for $L^{p(\cdot)}$, we get

$$
\int_{Q}|f(x)| d m=\int_{0}^{m Q} f^{*}(t) d t \leq\left\|f^{*}\right\|_{L^{p(\cdot)}}\|1\|_{L^{q(\cdot)}} \leq c_{Q}\|f\|_{\Lambda^{p(\cdot)}} .
$$

Let $w(t)$ be a nonnegative function defined on $[0, m \Omega]$ such that

$$
\left\|w^{-1}\right\|_{q(\cdot)}<\infty .
$$

Definition 3. The subset of all functions in $M(\Omega, m)$ for which

$$
\|f\|_{\Lambda_{w}^{p(\cdot)}}=\left\|w f^{* *}\right\|_{L^{p(\cdot)}}<\infty
$$

is called the space $\Lambda_{w}^{p(\cdot)}$.

If $w(t)=t^{\beta},-\frac{1}{p(0)}<\beta<\frac{1}{q(0)}$, then from Theorem IV it follows that

$$
\|f\|_{\Lambda_{w}^{p(\cdot)}} \approx\left\|f^{*} w\right\|_{\Lambda^{p(\cdot)}} .
$$

The space $\Lambda_{w}^{p(\cdot)}$ is a Banach space. The proof is similar as above.

In the sequel for $w(t)=t^{\beta}$ we put $\|\cdot\|_{\Lambda_{w}^{p(\cdot)}}=\|\cdot\|_{\Lambda_{\beta}^{p(\cdot)}}$.

\section{Integral transforms in $R^{n}$}

We begin with the mapping properties of singular operators

$$
K f(x)=V . P . \int_{R^{n}} \frac{k(y)}{|y|^{n}} f(x-y) d y, \quad x \in \Omega,
$$

in $\Lambda^{p(\cdot)}$, where $k$ is an odd function on $R^{n}$ homogeneous of degree 0 and satisfying the Dini condition on the unit sphere $S^{n-1}$ on $R^{n}$

$$
\int_{0}^{2} \frac{\omega(\delta)}{\delta} d \delta<\infty, \quad \text { where } \omega(\delta)=\sup _{x, y \in S^{n-1},|x-y| \leq \delta}|k(x)-k(y)| .
$$

As particular cases one may mention the Hilbert transform $(n=1$, $\left.k(x)=\frac{x}{|x|}\right)$ and the Riesz transforms $\left(n \geq 2, k(x)=\frac{x_{j}}{|x|}, j=1, \ldots, n\right)$.

Theorem 3.1. Let $1 \leq p(t)<P<\infty$ for $t \in[0, m \Omega]$. Let the conditions

$$
1<p_{0} \leq p(t)<P<\infty
$$

and

$$
\left|p\left(t_{1}\right)-p\left(t_{2}\right)\right| \leq \frac{A}{\ln \frac{1}{\left|t_{1}-t_{2}\right|}}, \quad\left|t_{1}-t_{2}\right| \leq \frac{1}{2}
$$

be satisfied in a neighbourhood $[0, d]$ of the origin, $d>0$. Then $K$ is bounded in $\Lambda^{p(\cdot)}$. 
Proof. As it is known (see [1])

$$
(K f)^{*}(t) \leq c\left(\frac{1}{t} \int_{0}^{t} f^{*}(y) d y+\int_{t}^{m \Omega} \frac{f^{*}(y)}{y} d y\right), \quad t>0 .
$$

Applying Theorem IV, we obtain the boundedness of $K$ in $L^{p(\cdot)}$.

Theorem 3.2. Let $p(t)$ satisfy the conditions of Theorem 2.1. Suppose that

$$
-\frac{1}{p(0)}<\beta<\frac{1}{q(0)}
$$

Then the inequality

$$
\|K f\|_{\Lambda_{\beta}}^{p}(\cdot) \leq c\|f\|_{\Lambda_{\beta}^{p(\cdot)}}
$$

holds with the constant $c$ not depending on $f$.

Proof. Applying (2.1) we obtain

$$
\begin{aligned}
(K f)^{*}(t) t^{\beta} & \leq c\left(t^{\beta-1} \int_{0}^{t} f^{*}(y) d y+t^{\beta} \int_{t}^{m \Omega} \frac{f^{*}(y)}{y} d y\right) \\
& =c\left(t^{\beta-1} \int_{0}^{t} \frac{f^{*}(y) y^{\beta}}{y^{\beta}} d y+t^{\beta} \int_{t}^{m \Omega} \frac{f^{*}(y) y^{\beta}}{y^{\beta+1}} d y\right) .
\end{aligned}
$$

Now from Theorem IV it follows that

$$
\left\|(K f)^{*} t^{\beta}\right\|_{L^{p(\cdot)}} \leq c\left\|f^{*} \cdot y^{\beta}\right\|_{L^{p(\cdot)}} .
$$

From Theorem 2.2, for the Riesz transforms

$$
R_{j} f(x)=V . P . \int_{\Omega} \frac{x_{j}-y_{j}}{|x-y|^{n+1}} f(y) d y, \quad j=1,2, \ldots, n,
$$

we have the following corollary.

Corollary 3.1. Let $p$ be as in Theorem 2.1. Then the operators $R_{j}$ $(j=1, \ldots, n)$ are bounded in $\Lambda_{\beta}^{p(\cdot)}$ under condition (2.2).

In the sequel we discuss the boundedness in $\Lambda^{p(\cdot)}$ of Riesz potentials and give an application to imbedding of certain spaces of differentiable functions.

The next theorem deals with the Riesz potential operator

$$
I_{\alpha} f(x)=\int_{\Omega} \frac{f(y)}{|x-y|^{n-\alpha}} d y, \quad x \in \Omega, \quad 0<\alpha<n .
$$

Theorem 3.3. Let $p(t)$ satisfy the assumptions of Theorem 2.2 and $s(x)$ be a measurable function on $[0, m \Omega]$ such that $1 \leq s(x)<S<\infty$ for all $x \in[0, m \Omega]$ and

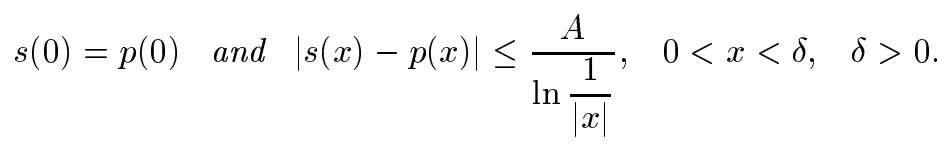


Then $I_{\alpha}$ is bounded from $L^{p(\cdot)}$ into $L^{s(\cdot)}$. Moreover, if

$$
-\frac{1}{p(0)}<\beta<\frac{1}{p(0)}
$$

then the inequality

$$
\left\|t^{\beta} I_{\alpha}\right\|_{\Lambda^{s(\cdot)}} \leq c\left\|t^{\beta} f\right\|_{\Lambda^{p(\cdot)}}
$$

holds with the constant $c$ not depending on $f$.

Proof. We make use of the estimate for the decreasing rearrangements of $I_{\alpha}$ (see [21]):

$$
\left(I_{\alpha} f\right)^{*}(t) \leq c\left(t^{-1+\alpha / n} \int_{0}^{t} f^{*}(y) d y+\int_{t}^{N} f^{*}(y) y^{-1+\alpha / n} d y\right)=c\left(B_{1} f+B_{2} f\right) .
$$

Applying Theorem IV, we obtain

$$
\begin{aligned}
\left\|B_{1} f\right\|_{L^{s(\cdot)}} & =\left\|t^{-1+\alpha / n} \int_{0}^{t} \frac{f^{*}(y) y^{\alpha / n}}{y^{\alpha / n}} d y\right\|_{L^{s(\cdot)}} \\
& \leq c\left\|f^{*}(y) y^{\alpha / n}\right\|_{L^{p(\cdot)}} \\
& \leq c\left\|f^{*}\right\|_{L^{p}(\cdot)} \\
& =c_{1}\|f\|_{\Lambda^{p(\cdot)}}
\end{aligned}
$$

since $0<t<m \Omega$ and $0<\alpha<n$.

Similarly, according to the same theorem we have

$$
\begin{aligned}
\left\|B_{2} f\right\|_{L^{s(\cdot)}} & =\left\|\int_{t}^{N} f^{*}(y) y^{-1+\alpha / n} d y\right\| \\
& \leq c\left\|f^{*}(y) y^{\alpha / n}\right\|_{L^{p(\cdot)}} \\
& \leq c_{1}\left\|f^{*}\right\|_{L^{p(\cdot)}} \\
& =c_{2}\|f\|_{\Lambda^{p(\cdot)}} .
\end{aligned}
$$

For a multi-index of nonnegative integers $K=\left(k_{1}, k_{2}, \ldots, k_{n}\right)$, let $D^{K}=\frac{\partial^{|K|}}{\partial_{1}^{k_{1}} \ldots \partial_{n}^{k_{n}}},|K|=k_{1}+k_{2}+\ldots+k_{n}$.

Theorem 3.4. Let $n \geq 2$ and let $k=|K|$ be any positive integer smaller than $n$. Suppose that $p(x)$ and $s(x)$ satisfy the assumptions of Theorem 2.1. Then

i) there exists a positive constant $c$ such that

$$
\|u\|_{\Lambda^{s(\cdot)}} \leq c\left\|D^{K} u\right\|_{\Lambda^{p(\cdot)}}
$$

for all real-valued functions $u$ on $\Omega$ where the continuation by zero beyond $\Omega$ has weak derivatives up to the order $k$ over $R^{n}$. 
(ii) If $\Omega$ is convex, then a positive constant $c$ exists such that

$$
\inf _{Q \in \mathcal{Q}_{k-1}}\|f-Q\|_{\Lambda^{s(\cdot)}} \leq c\left\|D^{K} u\right\|_{\Lambda^{p(\cdot)}}
$$

for all real valued functions $u$ on $\Omega$ which have weak derivatives up to the order $k$ in $\Omega$. Here $\mathcal{Q}_{k-1}$ denotes the set of all polynomials $Q$ of degree $\leq k-1$.

In the case $k=1$, inequality (2.5) holds with $Q$ equal to the mean value of $u$ over $\Omega, Q=\frac{1}{m \Omega} \int_{\Omega} u(x) d x$.

Proof. The part i). It is clear that $D^{K} u \in L^{1}(\Omega)$. Then by Theorem 1.1. $10 / 2$ of [20] we have the estimate

$$
|u(x)| \leq c \int_{\Omega} \frac{\left|D^{K} u(y)\right|}{|x-y|^{n-k}} d y \text { for } x \in \Omega .
$$

Applying Theorem 2.1, we arrive at the desired result.

The proof of part ii) is similar, since by Theorem 1.1. 10/1 of [9], there exists a constant $c$ depending only on $n, k$ and $\Omega$, and a polynomial $Q \in \mathcal{Q}_{k-1}$, depending on $u$, such that

$$
|u(x)-Q(x)| \leq c \int_{\Omega} \frac{\left|D^{K} u(y)\right|}{|x-y|^{n-k}} d y \text { for } x \in \Omega
$$

provided that $D^{K} u \in L^{1}(\Omega)$.

Now we pass to the mapping properties of Poisson integral and conjugate Poisson integrals in $\Lambda_{\beta}^{p(\cdot)}$ spaces. We consider the Poisson integral

$$
u_{f}(x, y)=\int_{\Omega} f(u) \frac{y}{\left(|x-u|^{2}+y^{2}\right)^{(n+1) / 2}} d u, \quad x, y \in \Omega
$$

and the system of conjugate Poisson integrals

$$
v_{f}^{j}(x, y)=\int_{\Omega} f(u) \frac{x_{j}-y_{j}}{\left(|x-u|^{2}+y^{2}\right)^{(n+1) / 2}} d u, x, y \in \Omega \quad j=1,2, \ldots, n .
$$

Since $m \Omega<\infty$, for $f \in L^{p(\cdot)}(\Omega)$ we have $f \in L^{p_{0}}(\Omega)$. Thus we conclude that

$$
T f(x)=\sup _{y>0}\left|u_{f}(x, y)\right| \leq c M f(x)
$$

and

$$
v_{f}^{j}(x, y)=u_{R_{j}}(x, y)
$$

(see [29], Chapters 6 and 2).

From (2.7), by the known estimate (see [1]) we have

$$
\left(\sup _{y}\left|u_{f}(x, y)\right|\right)^{*}(t) \leq c(M f)^{*}(t) \leq c_{1} 1 / t \int_{0}^{t} f^{*}(y) d y .
$$

By means of Theorem IV we derive the following result. 
Theorem 3.5. Let $p(t)$ and $\beta$ satisfy the conditions of Theorem I. Then $T$ is bounded in $\Lambda_{\beta}^{p(\cdot)}$.

Now consider the operator

$$
\widetilde{T}_{j} f(x)=\sup _{y}\left|v_{f}^{j}(x, y)\right| .
$$

By inequality (2.9) we have

$$
\left(\widetilde{T}_{j} f\right)^{*}(t) \leq c 1 / t \int_{0}^{t} f^{*}(y) d y .
$$

Thus

$$
\left\|\widetilde{T}_{j} f\right\|_{\Lambda_{\beta}^{p(\cdot)}} \leq c\left\|R_{j}\right\|_{\Lambda_{\beta}^{p(\cdot)}} .
$$

Basing on (2.10) and Corollary 1 we obtain the following result.

Theorem 3.6. Let a function $p(t)$ and a number $\beta$ satisfy the assumptions of Theorem 2.1. Then the operators $\widetilde{T}_{j}$ are bounded in $\Lambda_{\beta}^{p(\cdot)}$.

Remark 1. Applying the results of [5] and using the idea which was developed above, we can deduce that the theorems of this section are also valid in $R^{n}$ if a function $p(t)$ is assumed to satisfy the local logarithmic Dini condition and in addition is constant outside some large interval $\left(0, t_{0}\right)$, i.e. $p(t)=p, t>t_{0}$. For the power weight we assume that

$$
\max \left(-\frac{1}{p},-\frac{1}{p(0)}\right)<\beta<\min \left(\frac{1}{q}, \frac{1}{q(0)}\right),
$$

where $q=\frac{p}{p-1}$.

\section{Cauchy singular integrals on Lyapunov curves and curves of bounded rotation}

In this section we deal with the Cauchy singular integral

$$
S_{\Gamma} f(t)=\int_{\Gamma} \frac{f(\tau)}{\tau-t} d \tau, \quad t=t(s), \quad 0 \leq s \leq \ell,
$$

where $\Gamma$ is a finite rectifiable Jordan curve on which the arc-length is chosen as a parameter, starting from any fixed point.

$\Gamma$ is called Lyapunov curve if $t^{\prime}(s) \in \operatorname{Lip} \alpha, 0<\alpha \leq 1$. When $t^{\prime}(s)$ is a function of bounded variation, $\Gamma$ is called a curve of bounded rotation.

Our goal is to study the mapping properties of $S_{\Gamma}$ when $\Gamma$ is a Lyapunov curve or a curve of bounded rotation without cusps.

We assume the function $p(s)$ to be defined on $[0, l]$. The function $f(t(s))$ will be denoted by $f_{0}(s)$. 
Theorem 4.1. Let $\Gamma$ be a Lyapunov curve. The operator $S_{\Gamma}$ is bounded in $\Lambda^{p(s)}$ if

$$
1<p_{0} \leq p(s) \leq P<\infty \quad \text { for } \quad s \in[0, l]
$$

and the condition

$$
\left|p\left(s_{1}\right)-p\left(s_{2}\right)\right| \leq \frac{A}{\ln \frac{1}{\left|s_{1}-s_{2}\right|}}
$$

is satisfied in a neighbourhood of the origin.

Proof. In the case of Lyapunov curve the following estimate

$$
\left(S_{\Gamma} f\right)(t(\sigma)) \leq\left(\left|\int_{0}^{l} \frac{f_{0}(s)}{\sigma-s} d s\right|+\int_{0}^{l} \frac{\left|f_{0}(s)\right|}{|s-\sigma|^{1-\alpha}} d s\right)
$$

holds with $\alpha \in(0,1)$ (see e.g. [13]). Applying Theorems 2.1 and 2.3, we obtain the boundedness of $S_{\Gamma}$ in $\Lambda^{p(\cdot)}$.

Theorem 4.2. Let $\Gamma$ be a curve of bounded rotation without cusps. Let $p(s)$ satisfy the condition of Theorem 2.1 with $m$ denoting the arc-length measure on $\Gamma$. Then the operator $S_{\Gamma}$ is bounded in $\Lambda^{p(s)}$.

Proof. We have

$$
S_{\Gamma} f(t(s))=\int_{0}^{l} \frac{f_{0}(\sigma)}{\sigma-s} d \sigma+\int_{0}^{l}\left(\frac{t^{\prime}(\sigma) d \sigma}{t(\sigma)-t(s)}-\frac{1}{\sigma-s}\right) f_{0}(\sigma) d \sigma .
$$

As $t^{\prime}(s)$ is a function of bounded variation, we have

$$
\left|t^{\prime}(s)-t^{\prime}(\sigma)\right| \leq|V(s)-V(\sigma)|,
$$

where $V(s)$ is the total variation of $t^{\prime}$ on $[0, l]$.

Let

$$
T f(t(s))=\int_{0}^{l} h(\sigma, s) f_{0}(\sigma) d \sigma
$$

where

$$
h(\sigma, s)=\frac{t^{\prime}(\sigma)}{t(\sigma)-t(s)}-\frac{1}{\sigma-s} .
$$

Since $\Gamma$ is a curve of bounded rotation without cusps, it satisfies the arcchord condition, i.e.

$$
0<c_{1}<\left|\frac{t(s)-t(\sigma)}{s-\sigma}\right| .
$$

Therefore, we can derive the estimate

$$
\begin{aligned}
|h(\sigma, s)| & \leq\left|\frac{t^{\prime}(\sigma)}{t(\sigma)-t(s)}-\frac{1}{\sigma-s}\right| \\
& \leq \frac{c}{(s-\sigma)^{2}}\left|\int_{s}^{\sigma}\right| t^{\prime}(s)-t^{\prime}(u)|d u|
\end{aligned}
$$




$$
\begin{aligned}
& \leq \frac{c}{(s-\sigma)^{2}}\left|\int_{\sigma}^{s}\right| v(s)-v(u)|d u| \\
& \leq c(s-\sigma)^{-1}(V(s)-V(\sigma)) .
\end{aligned}
$$

Thus

$$
\begin{aligned}
|T f(t(s))| & \leq c \int_{0}^{l} \frac{V(s)-V(\sigma)}{s-\sigma} f_{0}(\sigma) d \sigma \\
& \leq c\left(V(s)\left|\int_{0}^{l} \frac{f_{0}(\sigma)}{s-\sigma} d \sigma\right|+\left|\int_{0}^{l} \frac{V(\sigma) f_{0}(\sigma)}{s-\sigma} d \sigma\right|\right) .
\end{aligned}
$$

Now from (3.1) and (3.3) and boundedness of the function $V$, in virtue of Theorem 1.1 we obtain the boundedness of $S_{\Gamma}$ in $\Lambda^{p(s)}$.

Note that for the $p(s)$ a constant function $p(s)=p$ the boundedness of $S_{\Gamma}$ on Lyapunov curve and on curve of bounded rotation without cusps was proved in [13] and [4], respectively.

Theorem 4.3. Let $\Gamma$ be a Lyapunov curve or a curve of bounded rotation without cusps. Let

$$
w(s)=|t(s)-t(0)|^{\beta}
$$

where

$$
-\frac{1}{p(0)}<\beta<\frac{1}{q(0)}
$$

Then the Cauchy singular operator $S_{\Gamma}$ is bounded in $\Lambda_{w}^{p}$.

Proof. As mentioned in the proof of Theorem 3.2, $\Gamma$ satisfies the arc-chord condition. Thus

$$
w(s) \sim s^{\beta} .
$$

Therefore, we may follow the scheme of the proof of Theorems 3.1 and 3.2 and apply Theorems 1.2 and 2.1 to obtain the boundedness of $S_{\Gamma}$ in $\Lambda_{w}^{p}$.

Basing on the recent results on the singular integrals from [7] and on the proofs of Theorems 3.1 and 3.2 we conclude the validity of the following theorem.

Theorem 4.4. Let $\Gamma$ be a Lyapunov curve or a curve of bounded rotation without cusps. If the function $p(s)$ satisfies the conditions (1.1) and (1.2) on $\bar{\Omega}=[0, l]$, then $S_{\Gamma}$ is bounded in $L^{p(s)}$.

Acknowledgements. This work was supported by Centro de Matemática Aplicada of Instituto Superior Técnico during the first author's visit to IST in February-March of 2002. 


\section{References}

[1] C. Bennett and K. Rudnik, On Lorentz-Zygmund spaces, Dissertationes Math. (Rozprawy Mat.), 175 (67) (1980).

[2] C. Bennett and R. Sharpley, Interpolation of Operators, Pure and Applied Mathematics, Vol. 129, Academic Press Inc., Boston, MA, 1988.

[3] D. Cruz-Uribe, A. Fiorenza and C.J. Neugebauer, The maximal function on variable $L^{p}$ spaces, Preprint, Istituto per le Applicazioni del Calculo "Mauro Picone" - Sezione di Napoli, (249).

[4] I. I. Danilyuk, Nonregular Boundary Value Problems in the Plane (Russian), Izdat. "Nauka", Moscow, 1975.

[5] L. Diening, Maximal functions on generalized Lebesgue spaces $L^{p}(x)$, Preprint, Mathematische Fakultät, Albert-Ludwigs-Universiẗ̈ Freiburg, (02).

[6] L. Diening, Maximal functions on generalized Lebesgue spaces $L^{p}(x)$, Math. Inequal. and Appl., (2002), to appear.

[7] L. Diening and M. Rüžička, Calderon-Zygmund operators on generalized Lebesgue spaces $L^{p}(x)$ and problems related to fluid dynamics, Preprint, Mathematische Fakultät, Albert-Ludwigs-Universität Freiburg, (21/2002, 04.07.2002) (2002), 1-20.

[8] D. E. Edmunds and A. Nekvinda, Averaging operators on $l^{\left\{p_{n}\right\}}$ and $L^{p}(x)$, Math. Inequal. Appl., 5 (2) (2002), 235-246.

[9] D.E. Edmunds, J. Lang and A. Nekvinda, On $L^{p(x)}$ norms, Proc. R. Soc. Lond., A, 455 (1999), 219-225.

[10] D.E. Edmunds and J. Rákosník, Density of smooth functions in $W^{k, p(x)}$, Proc. R. Soc. Lond., A, 437 (1992), 229-236.

[11] A. Fiorenza, A mean continuity type result for certain Sobolev spaces with variable exponent, Commun. Contemp. Math., 4(3) (2002), 587605.

[12] R. Hunt, On L( $p, q)$ spaces, L'Ens. Math., 12 (1966), 249-275.

[13] B.V. Khedelidze, The method of Cauchy type integrals for discontinuous boundary value problems of the theory of holomorphic functions of one variable (Russian), Itogi Nauki. i. Tekhniki, Sovren. Probl. Mat., 7 (1977), 5-162.

[14] V. Kokilashvili and S. Samko, Maximal and fractional operators in weighted $L^{p}(x)$ spaces, Revista Matematica Iberoamericana, to appear. 
[15] V. Kokilashvili and S. Samko, Maximal and fractional operators in weighted $L^{p}(x)$ - spaces, Proc. A. Razmadze Math. Inst., 129 (2002), $145-149$.

[16] V. Kokilashvili and S. Samko, Maximal and fractional operators in weighted $L^{p}(x)$ spaces, Preprint, Instituto Superior Técnico, Lisbon, Departamento de Matemática, (13) (2002), 1-26.

[17] V. Kokilashvili and S. Samko, Singular integrals and potentials in some Banach spaces with variable exponent, Preprint, Instituto Superior Técnico, Lisbon, Departamento de Matematica, (24), (2002), 1-14.

[18] V. Kokilashvili and S. Samko, Singular integrals and potentials in some Banach spaces with variable exponent, Preprint, Instituto Superior Técnico, Lisbon, Departamento de Matematica, (24) (2002), 1-14.

[19] O. Kovácík and J. Rákosník, On spaces $L^{p}(x)$ and $W^{k, p(x)}$, Czechoslovak Math. J., 41(116) (1991), 592-618.

[20] V. G. Maz'ja, Sobolev spaces, Springer Series in Soviet Mathematics, Springer-Verlag, Berlin, 1985.

[21] R.O. O'Neil, Convolution operators and $L^{(p, q)}$ spaces, Duke Math. J., 30 (1963), 129-142.

[22] M. Rüžička, Electroreological Fluids : Modeling and Mathematical Theory, Springer, Lecture Notes in Math., Vol. 1748 (2000), 176 pages.

[23] S.G. Samko, Convolution and potential type operators in $L^{p}(x)$, Integr. Transf. and Special Funct., 7 (3-4) (1998), 261-284.

[24] S.G. Samko, Differentiation and integration of variable order and the spaces $L^{p}(x)$, Proceed. of Intern. Conference "Operator Theory and Complex and Hypercomplex Analysis", 12-17 December 1994, Mexico City, Mexico, Contemp. Math., 212 (1998), 203-219.

[25] S.G. Samko, Denseness of $C_{0}^{1}\left(R^{n}\right)$ in the generalized Sobolev spaces $W^{m, p(x)}\left(R^{n}\right)$ (Russian), Dokl. Ross. Acad Nauk., 369(4) (1999), 451454 .

[26] I.I. Sharapudinov, The topology of the space $\mathcal{L}^{p(t)}([0,1])$ (Russian), Mat. Zametki, 26(4) (1979), 613-632.

[27] I.I. Sharapudinov, On the uniform boundedness in $L^{p}(p=p(x))$ of some families of convolution operators (Russian), Mat. Zametki, 59(2) (1996), 291-302.

[28] G. Sinnamon, Four Questions Related to Hardy Inequality, In : Function Spaces and Applications, Editors: D.E.Edmunds et. al., 255266. Alpha Science International Ltd, Pangbourne, UK, 2000. 
[29] E.M. Stein and G. Weiss, Introduction to Fourier Analysis on Euclidean Space (Russian). Moscow: Mir, 1974, 334 page. (English ed. in Princeton Univ. Press, 1971).
A. Razmadze Mathematical Institute
M. Aleksidze St.
1, 380093 Tbilisi
Georgia
(Email : kokil@rmi.acnet.ge)
Universidade do Algarve
Faro 8000
Portugal
(Email : ssamko@ualg.pt) 


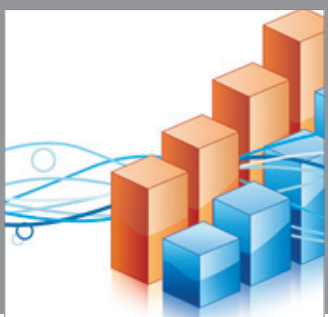

Advances in

Operations Research

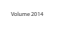

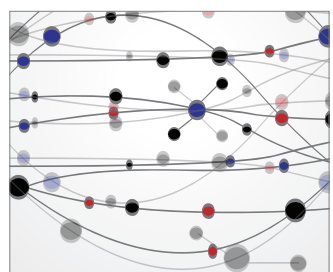

\section{The Scientific} World Journal
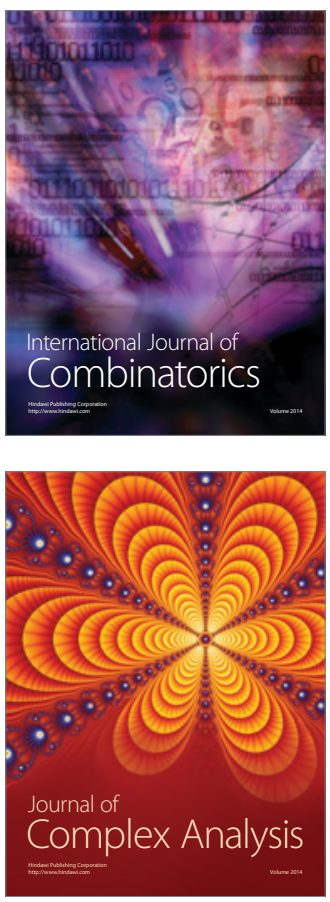

International Journal of

Mathematics and

Mathematical

Sciences
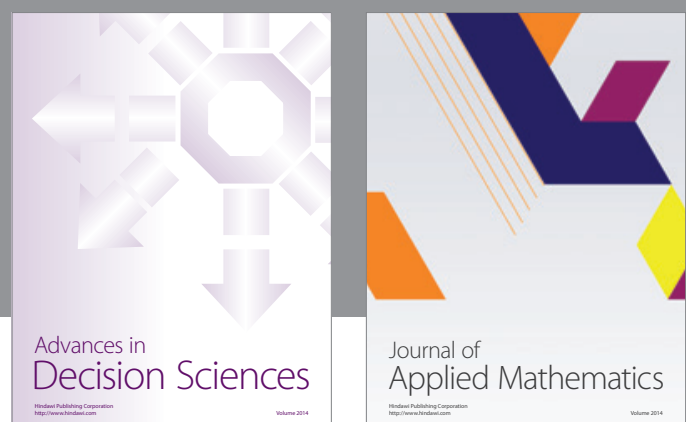

Journal of

Applied Mathematics
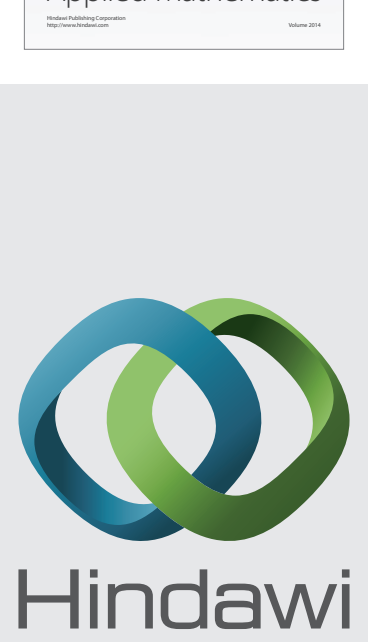

Submit your manuscripts at http://www.hindawi.com
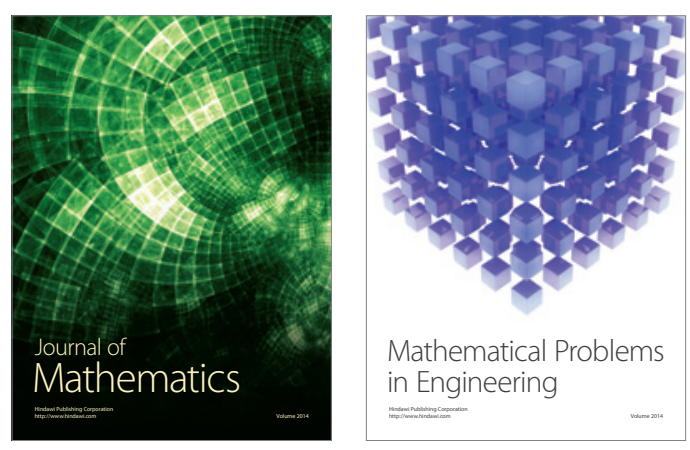

Mathematical Problems in Engineering
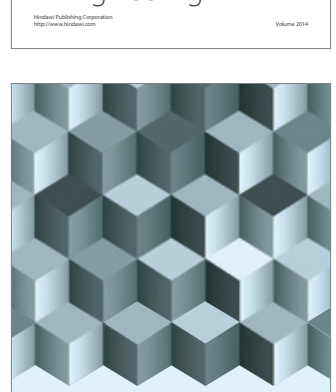

Journal of

Function Spaces
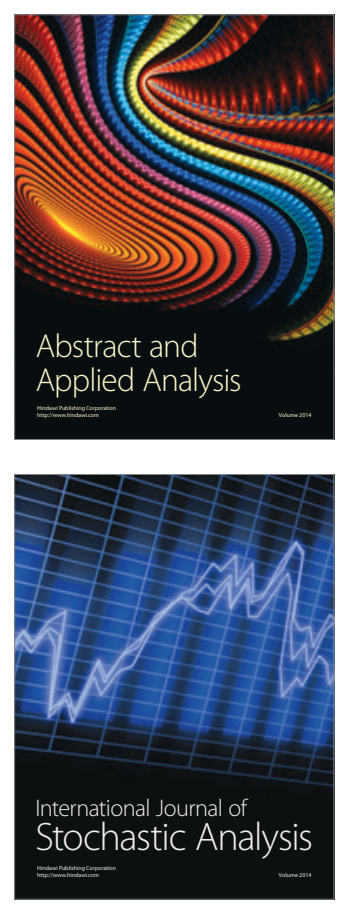

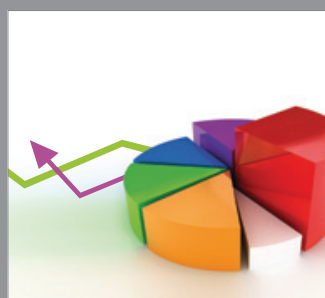

ournal of

Probability and Statistics

Promensencen
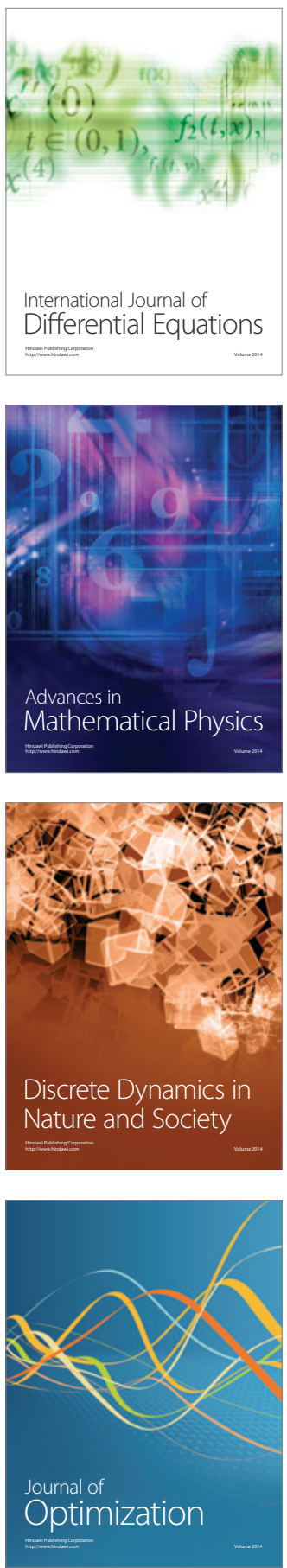